\title{
Non-Ischemic Remote Cardioprotective Phenomena
}

\section{Song Yang ${ }^{1}$ and Xiaoping Ren ${ }^{1,2,3 *}$}

${ }^{1}$ Hand and Microsurgical Center, Department of Orthopedics, The Second Affiliated Hospital of Harbin Medical University, China

${ }^{2}$ Clinical Translational Medicine Center, Harbin Medical University, Harbin, PR China

${ }^{3}$ Department of Pharmacology and Cell Biophysics, University of Cincinnati College of Medicine, Cincinnati, OH 45267-0575, USA.

\begin{abstract}
Ischemic preconditioning (IPC) is a powerful cardioprotective phenomenon that occurs in several species. IPC means that transient ischemic makes the cardioprotection in the subsequent period of ischemic thus it limits the scope of myocardial infarction. The reports of recent years have obtained the conclusion that this cardioprotection can be also induced by the non-ischemic preconditioning such as metabolic stimulation of myocardial cells and distention of the left ventricle. From the physiological point of view, the protective effect of non-ischemic preconditioning is associated with the higher proportion of myocardial cell metabolism. Remote ischemic preconditioning (RIC) is a therapeutic measure for cardioprotection against the deleterious effect of acute ischemia-reperfusion injury (IRI). And, it's beneficial effects are also seen in other organs (lung, liver, kidney, brain) and tissues (skeletal muscle). Although the mechanism of RIC has not yet been completely determined, there are several probable hypotheses supporting the cardioprotection induced by RIC, which include the neuronal pathway, humoral pathway, and systemic response. Recently, some researchers have provided experimental evidence for the cardioprotection under nonischemic trauma at a site from the heart produced by a transverse abdominal incision. This study demonstrated that protection could be elicited by non-ischemic stimulus which they term "remote preconditioning of trauma (RPCT)". Because of remote preconditioning of trauma, which is produced by an abdominal incision only through the skin, so it means that it's non-ischemic. Although remote ischemiac stimulus has been shown to elicit cardioprotection against IRI, there are not many reports about the remote non-ischemic stimulus and its mechanism. In this article, we provide a review of cardioprotection, the potential mechanism of RPCT as well as its clinical prospects.
\end{abstract}

Keywords: Cardioprotection; Remote preconditioning of trauma; Animal model; Potential underlying mechanism; Clinical application

\section{Introduction}

With the first described by Murry et al. [1] in 1986, Ischemic preconditioning (IPC) has been known for past two decades. IPC is a pretty powerful cardioprotective phenomenon that has been demonstrated that occurs in several species $[2,3]$ and there is strong evidence that it also occurs in humans [4]. However, IPC demands an absolutely intervention to be applied to the heart directly which may not be necessary in all clinical events. So in 1993, Przklenk et al. [5] showed the experimental evidence of remote preconditioning by demonstrating the protection in the left anterior coronary territory following a preconditioning stimulus in the circumflex coronary artery. Subsequently, some researchers termed this cardioprotective phenomenon "remote ischemic preconditioning (RIC)". Kharbanda et al. [6] introduced that transient limb which was induced by short periods of ischemia by tourniquet or blood pressure cuff had proven to be an uncomplicated method of inducing RIC. Meanwhile, the potential cardioprotection has been demonstrated in animal models as well as patients in Botker's report [7]. The protective phenomenon has been translated to positive clinical trials [8]. RIC is a therapeutic measure for cardioprotection against the deleterious effect of acute ischemia-reperfusion injury (IRI). Moreover, its beneficial effects are also seen in other organs (kidney, brain) and tissues (skeletal muscle) [9-11]. As for the mechanism of the RIC, some researchers have shown that remote preconditioning by transient limb ischemia in rabbits and humans is associated with release of a blood-borne, hydrophobic and small circulating factor, the effect of which can be blocked by the opiate receptor blocker, naloxone [12]. Subsequently, the same experimental group established the signaling of PI3K/Akt/GSK3 $\beta$ in the cardioprotection by the same stimulus of RIC [13]. In addition, it has been demonstrated that bradykinin (BK), adenosine, opioids, and norepinephrine (NE) all play an important role in RIC [14-16].
Some studies show that both IPC and RIC are associated with the activation of BK2R $[14,17]$. In brief, a variety of intracellular signaling mediators have been implicated in the protective effect of RIC such as G-protein cell surface coupled receptors, PKC, reactive oxygen release of circulating humoral factor(s), and activation of a systemic protective effect (such as an anti-apoptotic or anti-inflammatory response).

However, does remote non-ischemic preconditioning occur in animal or humans? This is an essential question from the clinical viewpoint meanwhile it could have the therapeutic implications $[18,19]$. Cardiac ischemia/reperfusion injury (IRI) contributes significantly to morbidity and mortality all over the world. Various researches for protecting the heart against myocardial infarction (MI) and IRI have been developed. In 2004, Ren et al. observed that infarct size after in vivo ischemia/reperfusion was altered by nonischemic surgical trauma. The transverse abdominal incision may result in the decreased infarct size in a TNFa-independent manner [20]. This study suggested that the cardioprotection could be induced by non-ischemicstimulus at a distance from the heart. To describe the phenomenon of this cardioprotection, they term it "remote preconditioning of trauma" (RPCT). In 2009, subsequently, the same group showed that the cardioprotective effect of RPCT is initiated by skin nociception, and requires neurogenic signaling involving spinal nerves and activation of cardiac sensory and

*Corresponding author: Xiaoping Ren, Department of Orthopedics, The Second Affiliated Hospital, Harbin Medical University, 194 Xuefu Rd, Nangang District, Harbin, China, Tel: 451-136-446-065-83; E-mail: renxg@uc.edu

Received May 20, 2013; Accepted June 03, 2013; Published June 07, 2013

Citation: Yang S, Ren X (2013) Non-Ischemic Remote

Cardioprotective Phenomena. Cardiol Pharmacol 2: 109. doi:10.4172/2329-6607.1000109

Copyright: (C) 2013 Yang S, et al. This is an open-access article distributed unde the terms of the Creative Commons Attribution License, which permits unrestricted use, distribution, and reproduction in any medium, provided the original author and source are credited. 
sympathetic nerves [21]. These results demonstrated that bradykinindependent activation and repression, respectively, of PKCe and PKCd in myocardium after RPCT, and their conclusion shows that the cardioprotection is associated with the $\mathrm{K}^{\mathrm{ATP}}$. The experiment suggests that nontraumatic nociciptive preconditioning represents a novel therapeutic strategy for cardioprotection with great potential clinical utility. Furthermore, in 2011, Gross et al. has showed that this protective phenomenon of heart in canine hearts [22]. Although it is found in canine hearts, it is significant for clinical setting and has implicated the cytochrome P450 epoxygenase pathway as a mediatory factor. This result suggests that bradykinin (BK) and the epoxyeicosatrienoic acids (EETs) share cardioprotective properties in a large animal model of RPCT. Recently, as a completely new concept, because RPCT could be mimicked non-invasively by topical application of capsaicin to induce the cardioprotection suggesting an innovative application without ischemia or trauma [23], since RPCT has been accepted by the researchers. At present, Ren's conference abstract expands the concept of RPCT again. Their experimental group tested the hypothesis that remote electrical stimulation (ES) of skin nociceptors could produce the effect similar to that of remote preconditioning of trauma. Mice $\mathrm{I} / \mathrm{R}$ model were established in vivo [24]. ES was used 15 minutes prior to $\mathrm{I} / \mathrm{R}$ or at the beginning of reperfusion. The results of research show that the skin nociceptor stimulation at specific points exactly reduce infarct size (85\% reduction), and they also find that cardioprotective effect of ES was abolished by both $\beta$-AR antagonism and in BK2R mice. Western blot results show PKC isoform translocation is altered after ES and pharmacologic blockade of PKC prevented cardioprotection due to ES. Therefore, they draw the conclusion 1) ES elicited a powerful cardioprotection via a neural mechanism. 2) The protective effect of ES against $\mathrm{MI}$ involves $\beta$-AR, BK2R signaling and PKC modulation. In other's experiment, the evidence was found that transcutaneous electrical nerve stimulation had been used widely clinically for nociceptive suppression by means of repeated C-fiber stimulation [25]. From remote cardioprotection described to the establishment of RPCT, there have been gone for past two decades. A variety of experimental models were set out to support the experiments for the powerful evidence. In the original study by Kharbanda and colleagues, transient ischemia of hind limb using a tourniquet was shown to reduce subsequent myocardial infarction (MI) size in pigs [6]. Subsequently, in experiment of introducing the RPCT and demonstrating the mechanism of RPCT by Ren and Jones group, a minimally traumatic mouse model was used to ascertain the effect of remote nonischemic surgical trauma upon I/R injury [20]. The abdominal incision used as the stimulus for RPCT was a $2-\mathrm{cm}$ transverse incision located on the abdominal midline. The incision extended through the skin and muscle and into the peritoneum and was immediately closed using 7-O polypropylene sutures. Kathrine's report showed that the rabbits were allocated several groups. Remote ischemic preconditioning was induced through four cycles of 5 minutes of hind limb ischemia (via a tourniquet). Some groups applied the $5 \mathrm{ml}$ of $0.1 \%$ capsaicin topical analgesic cream and other relative measures to demonstrate remote cardioprotection by direct peripheral nerve stimulation and topical capsaicin is mediated by circulating humoral factors [23]. In 2011, Garrett J Gross used the anesthetized adult mongrel dogs as the experimental model to demonstrate abdominal surgical incision induces RPCT via BK2R and the Cytochrome P450 Epoxygenase Pathway. Dogs were subjected to 60 minutes of left anterior descending coronary artery occlusion followed by 3 hours of reperfusion. A transverse slit $(9 \mathrm{~cm})$ was applied to abdominal wall of dogs to only slit the skin. The next year, the same group selected the rats to establish the animal model to show factors mediating remote preconditioning of trauma (the mechanism of cytochrome P450 epoxygenase pathway in mediating infarct size reduction). In their experiment, rats were randomly assigned to 30 groups for different treatments. In all studies, rats were subjected to 30 minutes of left coronary artery occlusion and 2 hours of reperfusion. The abdominal slit was performed 15 minutes prior to the 30 mints occlusion period. A $4-\mathrm{cm}$ transverse skin incision was made to induce RPCT. To sum up, the phenomenon of remote preconditioning of trauma can be induced in many species of animals. Thus, RPCT is a significant therapeutic measure, which laid the foundation for clinical application.

\section{Conclusion}

The discovery of remote cardioprotection has offered a significant therapeutic strategy for protecting the heart against the myocardial ischemia-reperfusion injury. The experimental research of past decade (especially RPCT) have encouraged scientists to do further exploration about the complex mechanism underlying the protective effect. Considering that the remote cardioprotection has been induced in many species of animals, the researchers should think about that how to combine the remote cardioprotection with the clinical work. This is the most important question from a clinical viewpoint, and could have therapeutic implications. In summary, remote cardioprotection especially remote non-ischemic preconditioning including RTCP is a pretty new field and could have clinical implications.

\section{References}

1. Murry CE, Jennings RB, Reimer KA (1986) Preconditioning with ischemia: a delay of lethal cell injury in ischemic myocardium. Circulation 74: 1124-1136.

2. Auchampach JA, Gross GJ (1993) Adenosine A1 receptors, KATP channels and ischemic preconditioning in dog. Am J Physiol 264: H1327-1336.

3. Goto M, Liu Y, Yang XM, Ardell JL, Cohen MV, et al. (1995) Role of bradykinin in protection of ischemic preconditioning in rabbit hearts. Circ Res 77: 611-621.

4. Kloner RA, Yellon D (1994) Does ischemic preconditioning occur in patients? J Am Coll Cardiol 24: 1133-1142.

5. Przyklenk K, Bauer B, Ovize M, Kloner RA, Whittaker P (1993) Regional ischemic 'preconditioning' protects remote virgin myocardium from subsequent sustained coronary occlusion. Circulation 87: 893-899.

6. Kharbanda RK, Mortensen UM, White PA, Kristiansen SB, Schmidt MR, et al (2002) Transient limb ischemia induces remote ischemic preconditioning in vivo. Circulation 106: 2881-2883.

7. Botker HE, Kharbanda R, Schmidt MR, Bottcher M, Kaltoft AK, et al. (2010) Remote ischaemic conditioning before hospital admission, as a complement to angioplasty, and effect on myocardial salvage in patients with acute myocardial infarction: a randomised trial. Lancet 375: 727-734.

8. Kharbanda RK, Nielsen TT, Redington AN (2009) Translation of remote ischaemic preconditioning into clinical practice. Lancet 374: 1557-1565.

9. Wever KE, Warlé MC, Wagener FA, van der Hoorn JW, Masereeuw R, et al (2011) Remote ischaemic preconditioning by brief hind limb ischaemia protects against renal ischaemia-reperfusion injury: the role of adenosine. Nephrol Dial Transplant 26: 3108-3117.

10. Jensen HA, Loukogeorgakis S, Yannopoulos F, Rimpiläinen E, Petzold A, et al. (2011) Remote ischemic preconditioning protects the brain against injury after hypothermic circulatory arrest. Circulation 123: 714-721.

11. Eberlin KR, McCormack MC, Nguyen JT, Tatlidede HS, Randolph MA, et al. (2009) Sequential limb ischemia demonstrates remote postconditioning protection of murine skeletal muscle. Plast Reconstr Surg 123: 8S-16S.

12. Shimizu M, Tropak M, Diaz RJ, Suto F, Surendra H, et al. (2009) Transient limb ischaemia remotely preconditions through a humoral mechanism acting directly on the myocardium: evidence suggesting cross-species protection. Clin Sci (Lond) 117: 191-200.

13. Li J, Xuan W, Yan R, Tropak MB, Jean-St-Michel E, et al. (2011) Remote 
preconditioning provides potent cardioprotection via PI3K/Akt activation and is associated with nuclear accumulation of $\beta$-catenin. Clin Sci (Lond) 120: 451 462.

14. Frances C, Nazeyrollas P, Prevost A, Moreau F, Pisani J, et al. (2003) Role of beta 1- and beta 2-adrenoceptor subtypes in preconditioning against myocardial dysfunction after ischemia and reperfusion. J Cardiovasc Pharmacol 41: 396405.

15. Zhou X, Fan GC, Ren X, Waggoner JR, Gregory KN, et al. (2007) Overexpression of histidine-rich Ca-binding protein protects against ischemia/ reperfusion-induced cardiac injury. Cardiovascular research 75: 487-497.

16. Ren XP, Wu J, Wang X, Sartor MA, Qian J, et al. (2009) MicroRNA-320 is involved in the regulation of cardiac ischemia/reperfusion injury by targeting heat-shock protein 20. Circulation 119: 2357-2366.

17. Kloner RA, Jennings RB (2001) Consequences of brief ischemia: stunning preconditioning, and their clinical implications: part2. Circulation 104: 31583167.

18. Hausenloy DJ, Yellon DM (2008) Remote ischaemic preconditioning: underlying mechanisms and clinical application. Cardiovasc Res 79: 377-386.

19. Heusch G, Musiolik J, Kottenberg E, Peters J, Jakob H, et al. (2012) STAT5 activation and cardioprotection by remote ischemic preconditioning in humans: short communication. Circ Res 110: 111-115.
20. Ren X, Wang Y, Jones WK (2004) TNF-alpha is required for late ischemic preconditioning but not for remote preconditioning of trauma. J Surg Res 121 120-129.

21. Jones WK, Fan GC, Liao S, Zhang JM, Wang Y, et al. (2009) Peripheral nociception associated with surgical incision elicits remote nonischemic cardioprotection via neurogenic activation of protein kinase C signaling. Circulation 120: S1-S9.

22. Gross GJ, Baker JE, Moore J, Falck JR, Nithipatikom K (2011) Abdomina surgical incision induces remote preconditioning of trauma (RPCT) via activation of bradykinin receptors (BK2R) and the cytochrome P450 epoxygenase pathway in canine hearts. Cardiovasc Drugs Ther 25: 517-522.

23. Redington KL, Disenhouse T, Strantzas SC, Gladstone R, Wei C, et al. (2012) Remote cardioprotection by direct peripheral nerve stimulation and topical capsaicin is mediated by circulating humoral factors. Basic Res Cardiol 107: 241.

24. Ren X (2013) A clinically relevant peripheral nociception stimulus elicits remote non-ischemic cardioprotection. The 3rd International Conference on Clinical \& Experimental Cardiology, April 15-17, 2013. Hilton Chicago/Northbrook, USA.

25. Sjolund BH (1988) Peripheral nerve stimulation suppression of C-fiber-evoked flexion reflex in rats. Part 2: Parameters of low-rate train stimulation of skin and muscle afferent nerves. J Neurosurg 68: 279-283. 